\title{
HAND2 is a novel obesity-linked adipogenic transcription factor regulated by glucocorticoid signalling
}

\author{
Maude Giroud ${ }^{1,2,3,4}$ - Foivos-Filippos Tsokanos ${ }^{1,2,3} \cdot$ Giorgio Caratti $^{5}\left(\mathbb{D} \cdot\right.$ Stefan Kotschi $^{4} \cdot$ Sajjad Khani $^{1,4}$ (D) $^{1,2,3}$ \\ Céline Jouffe ${ }^{1}$ (D) Elena S. Vogl ${ }^{1,2,3} \cdot$ Martin Irmler $^{6}$ (D) - Christina Glantschnig ${ }^{1,2,3}$ (D) - Manuel Gil-Lozano ${ }^{1,2,3}$ (D) \\ Daniela Hass $^{1,2,3}$. Asrar Ali Khan 1,2,3 (D) Marcos Rios Garcia ${ }^{1,2,3}$ (D) - Frits Mattijssen 1,2,3 - Adriano Maida ${ }^{1,2,3}$. \\ Daniel Tews $^{7}$ (D) Pamela Fischer-Posovszky ${ }^{7}$ (D) Annette Feuchtinger ${ }^{8}$ (D) Kirsi A. Virtanen $^{9} \cdot$ Johannes Beckers $^{6,2,10}$. \\ Martin Wabitsch ${ }^{7}$ (D) Henriette Uhlenhaut ${ }^{1,11}$ (D) - Matthias Blüher ${ }^{12}$ (D) Jan Tuckermann ${ }^{5}$ (D) \\ Marcel Scheideler ${ }^{1,2,3}$ (D) Alexander Bartelt ${ }^{1,4,13,14}$ (D) Stephan Herzig $^{1,2,3,15}$ (D)
}

Received: 14 November 2020 / Accepted: 18 February 2021 / Published online: 20 May 2021

(C) The Author(s) 2021

\begin{abstract}
Aims/hypothesis Adipocytes are critical cornerstones of energy metabolism. While obesity-induced adipocyte dysfunction is associated with insulin resistance and systemic metabolic disturbances, adipogenesis, the formation of new adipocytes and healthy adipose tissue expansion are associated with metabolic benefits. Understanding the molecular mechanisms governing adipogenesis is of great clinical potential to efficiently restore metabolic health in obesity. Here we investigate the role of heart and neural crest derivatives-expressed 2 (HAND2) in adipogenesis.

Methods Human white adipose tissue (WAT) was collected from two cross-sectional studies of 318 and 96 individuals. In vitro, for mechanistic experiments we used primary adipocytes from humans and mice as well as human multipotent adipose-derived stem (hMADS) cells. Gene silencing was performed using siRNA or genetic inactivation in primary adipocytes from loxP and or tamoxifen-inducible Cre-ERT2 mouse models with Cre-encoding mRNA or tamoxifen, respectively. Adipogenesis and adipocyte metabolism were measured by Oil Red O staining, quantitative PCR (qPCR), microarray, glucose uptake assay, western blot and lipolysis assay. A combinatorial RNA sequencing (RNAseq) and ChIP qPCR approach was used to identify target genes regulated by HAND2. In vivo, we created a
\end{abstract}

Alexander Bartelt

alexander.bartelt@med.uni-muenchen.de

Stephan Herzig

stephan.herzig@helmholtz-muenchen.de

1 Institute for Diabetes and Cancer (IDC); Helmholtz Zentrum München, German Research Center for Environmental Health, Neuherberg, Germany

2 German Center for Diabetes Research (DZD), Neuherberg, Germany

3 Joint Heidelberg-IDC Translational Diabetes Program, Inner Medicine 1, Heidelberg University Hospital, Heidelberg, Germany

4 Institute for Cardiovascular Prevention (IPEK), Ludwig-Maximilians-University, Munich, Germany

5 Institute for Comparative Molecular Endocrinology, Universität Ulm, Ulm, Germany

6 Institute of Experimental Genetics, Helmholtz Zentrum München, Neuherberg, Germany

7 Division of Pediatric Endocrinology and Diabetes, Department of Pediatrics and Adolescent Medicine, Ulm University Medical Center, Ulm, Germany
8 Research Unit Analytical Pathology, Helmholtz Center Munich, Neuherberg, Germany

9 Turku PET Centre, Turku University Hospital, Turku, Finland

10 Experimental Genetics, TUM School of Life Sciences, Technische Universität München, Freising, Germany

11 Metabolic Programming, TUM School of Life Sciences Weihenstephan and ZIEL Institute for Food \& Health, Munich, Germany

12 Helmholtz Institute for Metabolic, Obesity and Vascular Research (HI-MAG) of the Helmholtz Zentrum München at the University of Leipzig and University Hospital Leipzig, Leipzig, Germany

13 German Center for Cardiovascular Research (DZHK), Partner Site Munich Heart Alliance, Munich, Germany

14 Department of Molecular Metabolism, Harvard T.H. Chan School of Public Health, Boston, MA, USA

15 Molecular Metabolic Control, Medical Faculty, Technical University Munich, Munich, Germany 


\section{Research in context}

\section{What is already known about this subject?}

- HAND2, which is highly expressed in the heart, has been described to play a role in the differentiation of adipocyte stromal vascular fraction into cardiomyocyte-like cells

- HAND2 is differentially expressed and methylated between subcutaneous and visceral white adipose tissue in obese individuals

\section{What is the key question?}

- What is the role of HAND2 in adipogenesis?

What are the new findings?

- HAND2 is an obesity-linked adipocyte transcription factor

- HAND2 is regulated by glucocorticoids

- $\quad$ HAND2 is required for adipocyte differentiation in vitro

\section{How might this impact on clinical practice in the foreseeable future?}

- Understanding the molecular mechanisms governing adipogenesis is of great clinical potential to efficiently restore metabolic health in obesity

conditional adipocyte Hand 2 deletion mouse model using Cre under control of the Adipoq promoter (Hand2 $2^{\text {AdipoqCre }}$ ) and performed a large panel of metabolic tests.

Results We found that HAND2 is an obesity-linked white adipocyte transcription factor regulated by glucocorticoids that was necessary but insufficient for adipocyte differentiation in vitro. In a large cohort of humans, WAT HAND2 expression was correlated to BMI. The HAND2 gene was enriched in white adipocytes compared with brown, induced early in differentiation and responded to dexamethasone (DEX), a typical glucocorticoid receptor (GR, encoded by $N R 3 C 1$ ) agonist. Silencing of $N R 3 C 1$ in hMADS cells or deletion of GR in a transgenic conditional mouse model results in diminished HAND2 expression, establishing that adipocyte HAND2 is regulated by glucocorticoids via GR in vitro and in vivo. Furthermore, we identified gene clusters indirectly regulated by the GR-HAND2 pathway. Interestingly, silencing of HAND2 impaired adipocyte differentiation in hMADS and primary mouse adipocytes. However, a conditional adipocyte Hand2 deletion mouse model using Cre under control of the Adipoq promoter did not mirror these effects on adipose tissue differentiation, indicating that HAND2 was required at stages prior to Adipoq expression.

Conclusions/interpretation In summary, our study identifies HAND2 as a novel obesity-linked adipocyte transcription factor, highlighting new mechanisms of GR-dependent adipogenesis in humans and mice.

Data availability Array data have been submitted to the GEO database at NCBI (GSE148699).

Keywords Adipocytes $\cdot$ Dexamethasone $\cdot$ Differentiation $\cdot$ Glucocorticoid receptor $\cdot$ HAND2 $\cdot$ hMADS $\cdot$ Human adipose tissue $\cdot$ Mesenchymal stem cells $\cdot$ Obesity $\cdot$ Transcription factor

\section{Abbreviations}

BAT Brown adipose tissue

DEX Dexamethasone

DIO Diet-induced obesity

GC Glucocorticoid

GR Glucocorticoid receptor

gWAT Gonadal white adipose tissue

HAND2 Heart and neural crest derivatives-expressed 2

HFD High-fat diet

hMADS Human multipotent adipose-derived stem (cell)
hSVF Human stromal vascular fraction

mSVF Mouse stromal vascular fraction

PPAR $\gamma \quad$ Peroxisome proliferator-activated receptor $\gamma$

qPCR Quantitative PCR

RNASeq RNA sequencing

scWAT Subcutaneous white adipose tissue

SVF Stromal vascular fraction

visWAT Visceral white adipose tissue

WAT White adipose tissue 


\section{Introduction}

Adipocytes are specialised cells that have a variety of functions including nutrient buffering, endocrine regulation and thermogenesis. Principally, there are two types of adipocytes: first, white adipocytes, able to store and release lipids and secrete adipokines and, second, thermogenic adipocytes (brown and beige), which additionally dissipate chemical energy from nutrients as heat [1]. Obesity, the excess accumulation of white adipose tissue (WAT), is characterised by adipocyte dysfunction and metabolic imbalance. Enhancing adipocyte health or increasing the number of functional adipocytes has beneficial effects on systemic metabolism.

Adipogenesis is a complex process regulated by the interplay of transcription factors, metabolites and hormonal cues. The commitment of mesenchymal stem cells to becoming preadipocytes and adipogenesis occur in waves of transcriptional programs. Genes involved in development, for example those encoding bone morphogenic proteins, Wnt or hedgehog proteins, play a critical role during the commitment of progenitor cells to the adipocyte lineage [2] while activating peroxisome proliferator-activated receptor $\gamma$ (PPAR $\gamma$ ), for example by rosiglitazone, promotes later adipocytes differentiation and induces a thermogenic phenotype [3].

Glucocorticoids (GCs) are steroid hormones modulating metabolism [4-6] including adipogenesis, lipolysis, lipogenesis and thermogenesis [7]. However, recent studies have demonstrated that the glucocorticoid receptor (GR) is not required for the development of adipose tissue in mouse models [8-11]. Nevertheless, the effects of GCs on adipocytes are multifaceted and largely depend on the metabolic status of the individual. Chronically elevated levels of GCs, either by pharmacological treatment or in patients with Cushing's syndrome, results in partial lipodystrophy [12]. However, neither the adipose-specific activation of GR by GCs nor their regulation in obesity are fully understood.

In search of novel adipose-specific mechanisms linked to human obesity we have previously investigated methylation and gene expression signatures of visceral WAT (visWAT) and subcutaneous WAT (scWAT) and found that the transcription factor HAND2 (heart and neural crest derivatives-expressed 2 ) is among the most differentially expressed genes [13]. $H A N D 2$ encodes a helix-loop-helix transcription factor that plays a role in cardiac morphogenesis by binding other helix-loophelix proteins including its close relative HAND1 [14]. This ability of HAND2 to control organ development [15-18] and cell differentiation led us to hypothesise that HAND2 might be involved in adipocyte differentiation and function.

\section{Methods}

For detailed methods, please refer to the Electronic supplementary material (ESM).
Cell culture and stromal vascular fraction preparation Human multipotent adipose-derived stem (hMADS) cells were kindly provided by E.Z. Amri. hMADS were free of mycoplasma contamination and cultivated as previously described $[19$, 20]. Mouse stromal vascular fraction (SVF; mSVF) was isolated from brown adipose tissue (BAT), scWAT, and visWAT, and differentiated into adipocytes [21]. Human SVF (hSVF) was isolated from scWAT, collected from healthy patients (abdominoplasty) and differentiated by administering the same adipogenic cocktail as for hMADS [21]. The study was approved by the University of Ulm Ethics Committee (vote no. 300/16) and all donors gave written informed consent.

Gene expression and functional analysis in vitro HAND2 and NR3C1 mRNA was silenced in hSVF, hMADS and mSVF using $20 \mathrm{nmol} / 1$ siRNA (SMARTpool ON-TARGETplus siRNA Horizon Discovery, UK), cells were collected $48 \mathrm{~h}$ after transfection. To overexpress Hand2 we infected mSVF or 3T3-L1 cells with the pcDNA-3XFlag-Hand2 vector [15] and the pENTR-CMV-MCS-TKpA as control using TransITX2 Dynamic Delivery System (Mirus, USA, MIR 6003). Cells were collected $72 \mathrm{~h}$ after infection. Chemical activation and inhibition of GR were performed by $12 \mathrm{~h}$ treatment with respectively $1 \mu \mathrm{mol} / 1$ dexamethasone (DEX) (Merck, Germany D4902) and/or 2 mol/l RU486 (475838, Merck). Transcriptional analysis was performed using SYBR-based quantitative PCR (qPCR) (primers listed in ESM Table 1), microarray (Human Clariom S arrays, Thermo Fisher Scientific, Germany) and RNAseq (performed by Novogene, UK). Protein levels of Akt, pAkt and $\beta$-actin (Cell Signaling, Germany \#9272, \#4051, \#4970, respectively, 1/1000) were quantified by western blot [22]. Oil Red O staining, glycerol accumulation and glucose uptake was measured. ChIP qPCR was performed as previously described [23].

Mouse experiments All animal studies were conducted in accordance with German animal welfare legislation and protocols were approved by the state Ethics Committee and Government of Upper Bavaria (nos. ROB-55.22532.Vet_02-16-117; ROB-55.2-2532.Vet_02-17-125; ROB-55.2-2532.Vet_02-15-164). All mice were grouphoused and maintained in a climate-controlled environment at $22^{\circ} \mathrm{C}$ with a $12 \mathrm{~h}$ dark-light cycle under specific pathogen-free conditions in the animal facility of the Helmholtz Center Munich. $d b / d b$ mice (JAX mouse strain) were purchased from Charles River (https://www.criver. $\mathrm{com} /$ products-services/find-model/jax-dbdb-mice?region= 23 ) Adipocyte-specific Hand 2 knockout mice (Hand2 ${ }^{\text {AdipoqCre }}$ ) were generated by crossing Adipo ${ }^{\mathrm{CRE}}$ mice (Jackson laboratory, stock number 028020; C57BL/6J, https://www.jax.org/strain/028020) with Hand2 $2^{\text {flox/flox }}$ mice (NMRI strain), kindly provided by R. Zeller [24]. 
Hand2 $2^{\text {AdipoqCre }}(\mathrm{CRE}+)$ and wild-type littermates (CRE-) were used for all experiments. Animals were fed a high-fat diet (HFD) 60\% energy from fat (Research Diets, New Brunswick, NJ, USA D12492) ad libitum from the age of 6 weeks for 12 weeks, after which glucose and insulin tolerance tests were performed. Briefly, animals were fasted and subsequently injected intraperitoneally with glucose at $2 \mathrm{~g} / \mathrm{kg}$ or insulin $0.8 \mathrm{U} / \mathrm{kg}$. Body mass composition measurement, necropsy, and haematoxylin/eosin (HE) staining were performed. Histological staining with HE was performed on $3 \mu \mathrm{m}$ thick sections of BAT, scWAT and gWAT. Intraperitoneal injection of DEX (1 $\mathrm{mg} / \mathrm{kg})$ (Sigma, Germany \#D9184-5G) was performed on wild-type C57BL/ 6J (JAX mouse strain) (Charles River, https://www.criver. com/products-services/find-model/jax-c57bl6j-mice) for $6 \mathrm{~h}$, 2 days or 2 weeks and Hand2 ${ }^{\text {AdipoqCre }}$ for $6 \mathrm{~h}$. Indirect calorimetry, including energy expenditure, food consumption, oxygen consumption and locomotor activity, was measured for Hand $2^{\text {AdipoqCre }}$ using metabolic cages (TSE PhenoMaster cages TSE Systems, Bad Homburg, Germany). $G R^{\text {flox/flox }}$ and $G R^{\text {ERT2Cre }}$ (on a C57BL/6 background) and Hand2 $2^{3 \mathrm{XFlag}}$ (on an NMRI background) mice were generated as previously described $[15,25]$. Hand 2 expression was analysed using SYBR-based qPCR (Thermo Fisher, Germany \#A25741) (primers listed in ESM Table 1).

Human studies The study protocol relating to human BAT was approved by the Ethics Committee of the Hospital District of Southwestern Finland, and participants provided written informed consent following the committee's instructions. The study was conducted according to the principles of the Declaration of Helsinki. Human BAT was collected from fluorodeoxyglucose F18-positron emission tomographypositive scan areas in the supraclavicular location and scWAT was taken from the same incision. The studies referring to the human cohort 1 and 2 were approved by the Ethics Committee of the University of Leipzig (approval no: 159-1221052012) and performed in accordance to the declaration of Helsinki. All participants gave written informed consent before taking part in the study. Human cohort 1 refers to human scWAT versus visWAT samples collected in the context of a cross-sectional study of 318 individuals (249 women, 69 men; BMI range: $21.9-97.3 \mathrm{~kg} / \mathrm{m}^{2}$, age range: 19-75 years). An additional 13 individuals receiving DEX treatment for chronic inflammatory diseases were compared to BMI-matched $\left(35-67 \mathrm{~kg} / \mathrm{m}^{2}\right)$ individuals from cohort 1 . In cohort 2, 96 individuals were selected from the Leipzig Obesity Biobank to define age- and sex-matched groups of healthy lean individuals ( $n=32$; mean BMI: $23.4 \pm 1.5 \mathrm{~kg}$ / $\mathrm{m}^{2}$ mean age $43.6 \pm 7.1$ years, 23 female, 9 male participants), individuals with metabolically healthy obesity $(n=32$; mean BMI: $45.9 \pm 6.8 \mathrm{~kg} / \mathrm{m}^{2}$ mean age $42.6 \pm 9.3$ years, 23 female, 9 male participants) further BMI-matched to 32 individuals with obesity and type 2 diabetes (mean BMI: $45.3 \pm 4.7 \mathrm{~kg}$ / $\mathrm{m}^{2}$ mean age $42.7 \pm 6.7$ years, 25 female, 7 male participants).

HAND2 mRNA expression was measured using SYBRbased qPCR (primers listed in ESM Table 1).

Statistics Data presented as bar charts were expressed as mean \pm SEM. Data presented as box and whisker plots were shown as median with upper and lower quartile \pm maximum and minimum points. Two-tailed, unpaired $t$ test was used when comparing two conditions. One-way ANOVA and two-way ANOVA with Tukey test were used when comparing three or more groups, as reported in the figure legends. Analysis was performed using GraphPad Prism. A $p$ value $<0.05$ was considered significant as indicated by asterisks in the figure legends. Mouse experiments involving Hand2 $2^{\text {AdipoqCre }}$ were performed three times independently and pooled. Mice were excluded for poor body condition or if they died before the end of the experiment. For the quantification of HAND2 in control patients versus DEX-treated patients, only patients in the same range of BMI were considered, and outliers were excluded using the ROUT test Q1\% from GraphPad Prism.

\section{Results}

Adipose HAND2 is correlated to obesity in mice and humans In order to investigate the importance of HAND2 in adipose tissue biology, we first determined its gene expression in different adipose depots obtained from groups of non-obese, obese and diabetic individuals over a broad range of BMI. HAND2 mRNA levels were higher in visWAT compared with scWAT (cohort 1) (ESM Fig. 1a) and were lower in obese or diabetic participants compared with lean participants, especially in the visWAT (cohort 1) (Fig. 1a). We confirmed these findings in a second independent cohort (cohort 2) consisting of an equal number of lean, obese and diabetic participants (ESM Fig. 1b). ScWAT depots showed substantially higher HAND2 expression than human BAT (Fig. 1b). Intriguingly, HAND2 expression in visWAT but not in scWAT was inversely correlated with BMI in these patients (Fig. 1c,d). However, HAND2 in both tissues was correlated with body weight in both cohorts (ESM Table 2). In line with these human data, in mice Hand2 expression was, as expected, highest in the heart followed by liver and gonadal WAT (gWAT), with lower expression in scWAT, BAT and gastrocnemius muscle (ESM Fig. 1c). Moreover, gWAT Hand2 was significantly lower in mouse models of obesity, including HFD-induced obesity (referred to here as diet-induced obesity [DIO]) as well as genetically obese, leptin receptor-deficient $d b / d b$ mice (Fig. 1e,f), and also inversely correlated with body weight (Fig. 1g,h). These data demonstrate that HAND2 was prominently expressed in gWAT and correlated with obesity in both mice and humans. 
Fig. 1 White adipocyte HAND2 is correlated to obesity in mice and humans. (a, b) HAND2 expression in visWAT vs scWAT from lean, obese or diabetic participants ( $n=318$ participants; cohort 1) (a), and in human scWAT vs BAT $(n=7$ individuals) (b). (c, d) HAND2 expression correlated with BMI in $\operatorname{visWAT}(\mathbf{c})$ and $\operatorname{scWAT}(\mathbf{d})(n=$ 318 participants; cohort 1). (e, f) Hand 2 expression in wild-type vs DIO mice $(n=10$ mice $)(\mathbf{e})$ and in WT vs $d b / d b$ mice ( $n=5$ mice) (f). (g, h) Hand2 expression correlated with body weight in mouse gWAT (g) and scWAT (h) ( $n=54$ mice). Data are presented as arbitrary units representing copy number of HAND2 normalised to HPRT1 (a, $\mathbf{c}, \mathbf{d})$ or $\operatorname{Tbp}(\mathbf{g}, \mathbf{h})$; as fold change compared to scWAT (b); or as fold change compared to gWAT/WT (e, f). Statistics: oneway ANOVA with Tukey test (a), two-tailed paired $t$ test (b), correlation (c, d, $\mathbf{g}, \mathbf{h}$ ), two-way ANOVA with Tukey test (e, f). Data are presented as median with upper and lower quartile \pm maximum and minimum $(\mathbf{a}, \mathbf{b}, \mathbf{e}$, f). Statistical significance is indicated by $* p<0.05$. A.U., arbitrary units; T2D, type 2 diabetes; WT, wild-type a
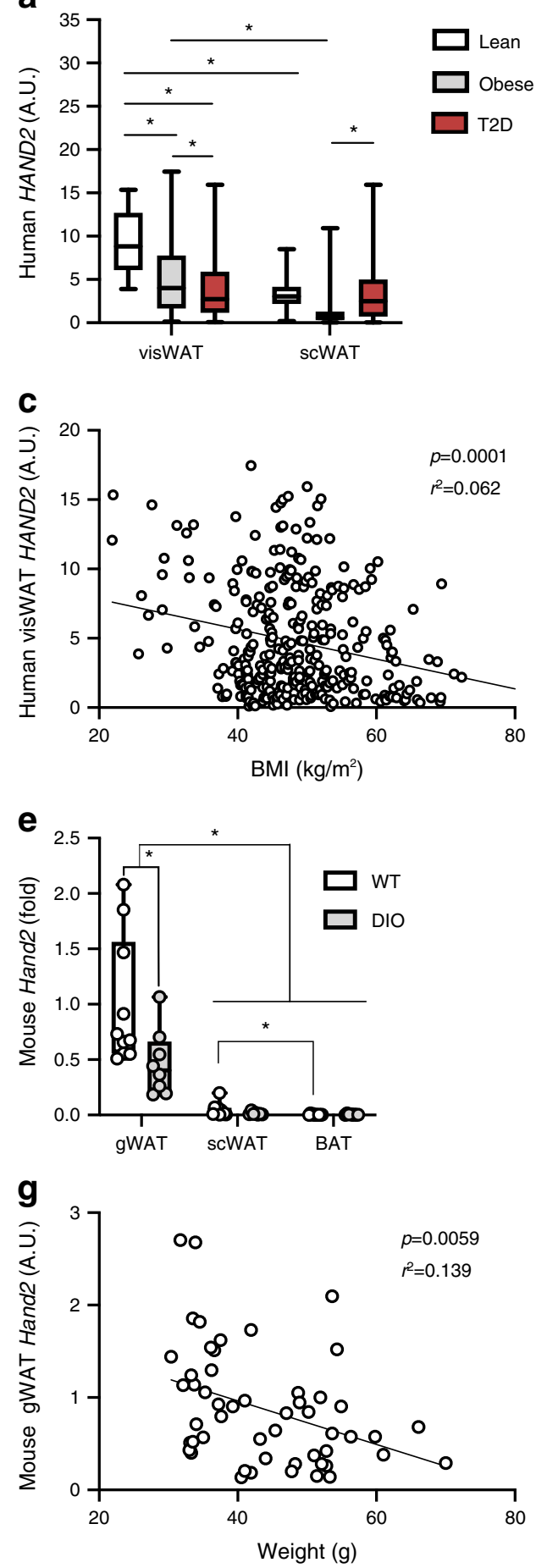

b
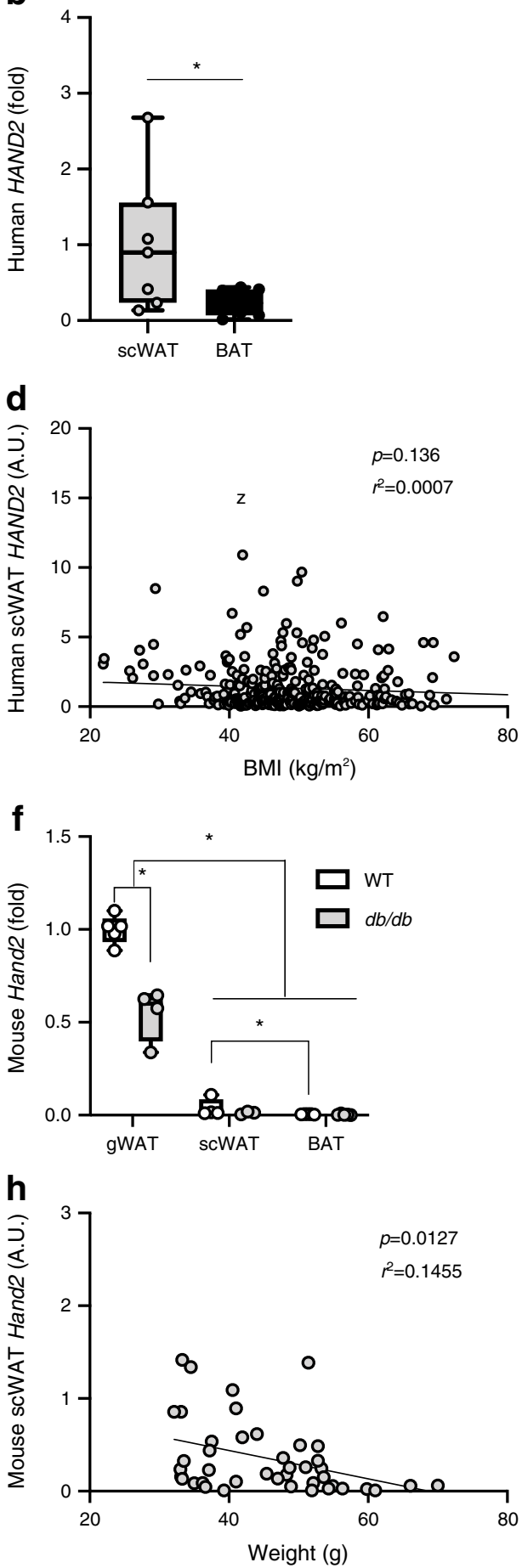

HAND2 is expressed in adipocytes and is induced early in adipogenesis The cellular composition of adipose tissue is heterogenous and changes dynamically in obesity. To analyse the origin of HAND2 expression in more detail, we next measured HAND2 mRNA levels in fractionated adipose tissue from mouse and human samples ex vivo. Mouse Hand2 was poorly expressed in the macrophage fraction and its levels were not significantly higher in mSVF compared with the adipocyte fraction $(p=0.2)$ (Fig. 2a,b) while in human adipose tissue, HAND2 levels were higher in the hSVF compared with the adipocyte fraction (Fig. 2c). These findings indicate that HAND2 was predominantly expressed in preadipocytes and adipocytes. To further evaluate this concept, we employed in vitro adipocyte models. Differentiated 

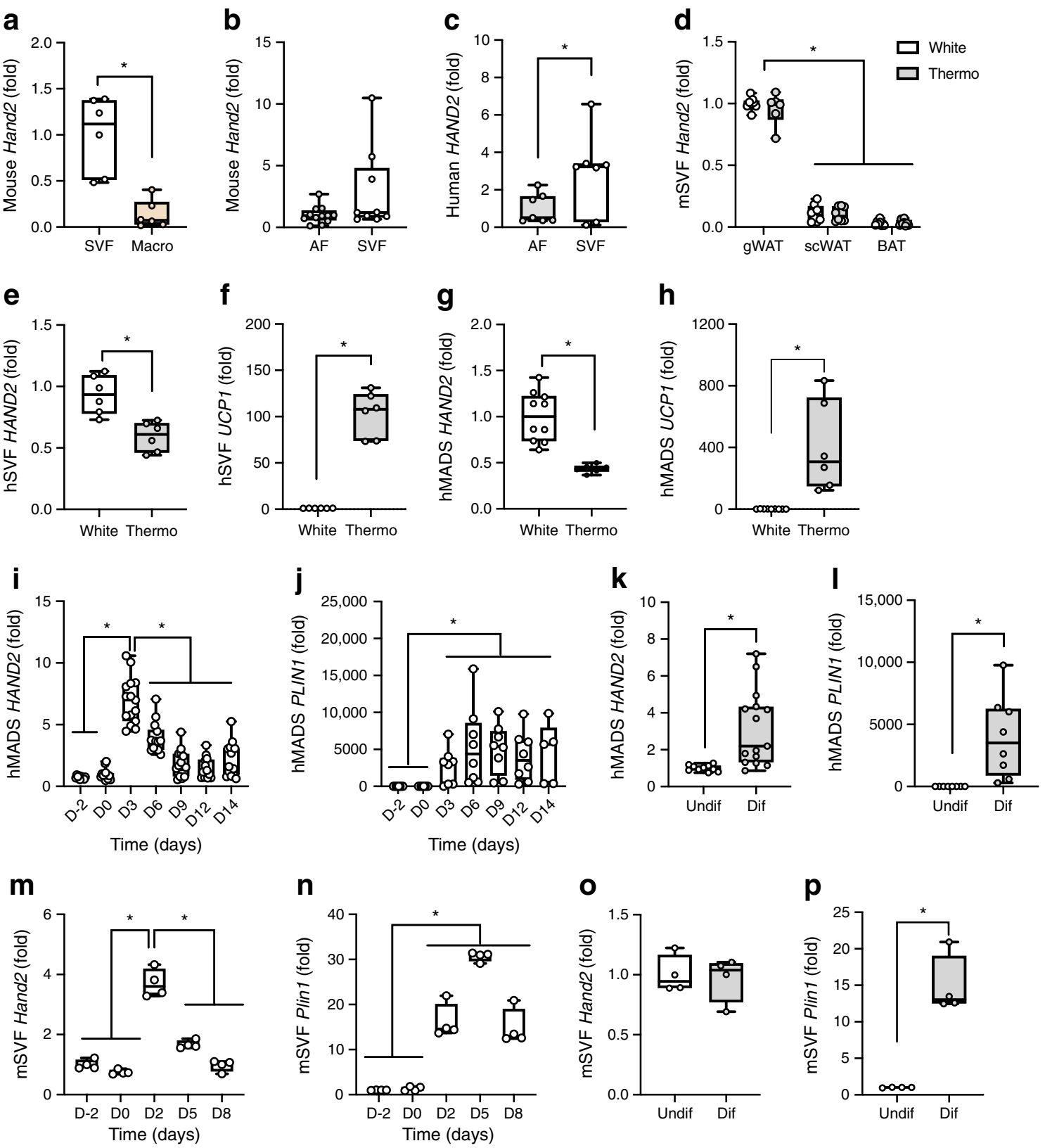

Fig. 2 HAND2 is induced in early differentiation and expressed in both pre-adipocytes and mature adipocytes. (a-c) Hand 2 expression in $\mathrm{mSVF}$ vs macrophages ( $n=6$ replicates) (a), vs adipocyte fraction (AF $n=12$ replicates, SVF $n=9$ replicates) (b) and in human $\mathrm{AF} \operatorname{vs~hSVF~(~} n=7$ replicates) (c). (d) Hand2 expression in mSVF from different fat depots differentiated using rosiglitazone into white or thermogenic adipocytes ( $n=8$ replicates). (e, f) HAND2 (e) and $U C P 1$ (f) expression in hSVF differentiated into adipocytes (White $n=9$ replicates, Thermo $n=6$ replicates). (g, h) HAND2 (g) and UCP1 (h) expression in hMADS mature adipocytes (White $n=10$ replicates, Thermo $n=6$ replicates). (i, j) HAND2 (i) and PLIN1 (j) expression in hMADS cells during differentiation ( $n=9-15$ replicates). (k, l) HAND2 (k) and PLIN1 (l) expression in hMADS pre-adipocytes and mature adipocytes $(n=12$ replicates). $(\mathbf{m}, \mathbf{n})$

adipocytes derived from the mSVF of distinct anatomical locations mirrored the in vivo Hand 2 mRNA expression pattern with highest levels in adipocytes from gWAT
Hand2 (m) and Plin1 (n) expression during mSVF differentiation $(n=4$ replicates). (o, p) Hand2 (o) and Plin1 (p) expression in mSVF preadipocytes and mature adipocytes ( $n=4$ replicates). Data are presented as fold change compared to the condition $\operatorname{mSVF}(\mathbf{a})$; to the condition $\mathrm{AF}$ $(\mathbf{b}, \mathbf{c})$; to the condition gWAT/White (d); to the condition White (e-h); to the condition D-2 (i, j, m, n); to the condition Undif $(\mathbf{k}, \mathbf{l}, \mathbf{o}, \mathbf{p})$. Statistics: two-tailed unpaired $t$ test $(\mathbf{a}, \mathbf{b}, \mathbf{c}, \mathbf{e}, \mathbf{f}, \mathbf{g}, \mathbf{h}, \mathbf{k}, \mathbf{l}, \mathbf{o}, \mathbf{p})$; one-way ANOVA with Tukey test $(\mathbf{i}, \mathbf{j}, \mathbf{m}, \mathbf{n})$, two-way ANOVA with Tukey test (d); median with upper and lower quartile \pm maximum and minimum (a-p). Statistical significance is indicated by $* p<0.05$. AF, adipocyte fraction; D, day; Dif, differentiated; Macro, macrophages; Thermo, thermogenic; Undif, undifferentiated

compared with those isolated from scWAT or from BAT (Fig. 2d). In light of the established role of HAND2 in development and its high expression in SVF, we investigated 
a

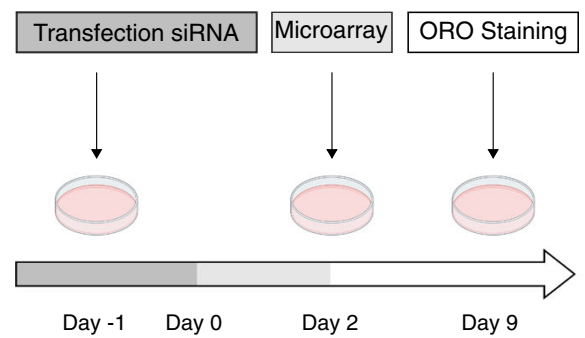

d

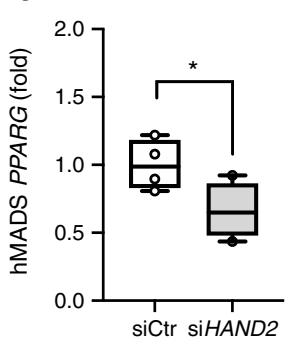

e

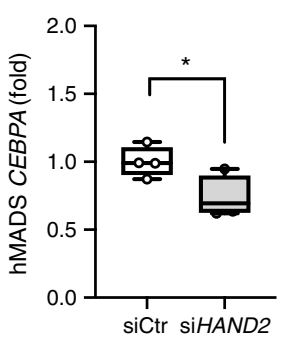

b $\square$ siCtr $\square$ siHAND2 C

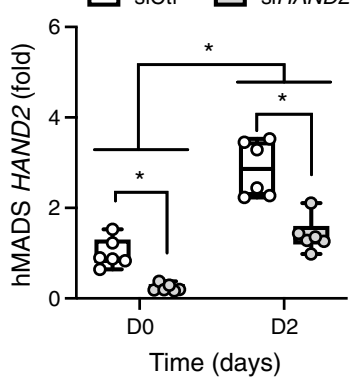

f

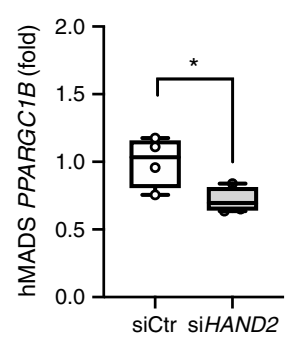

C

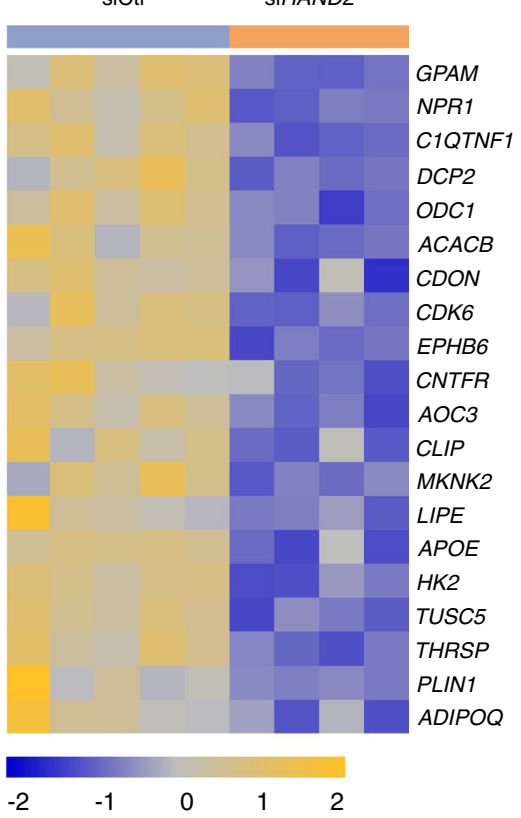

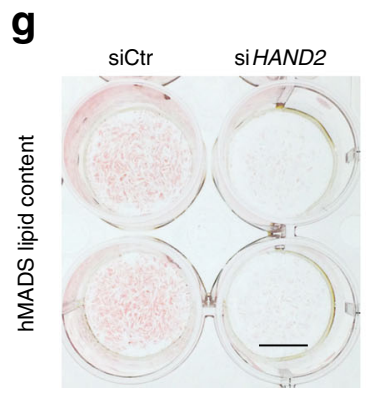

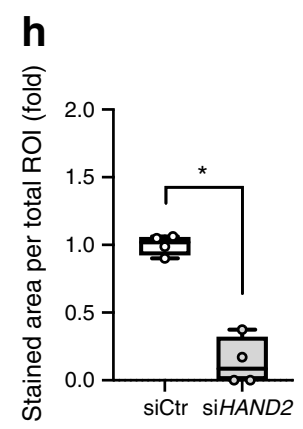

i
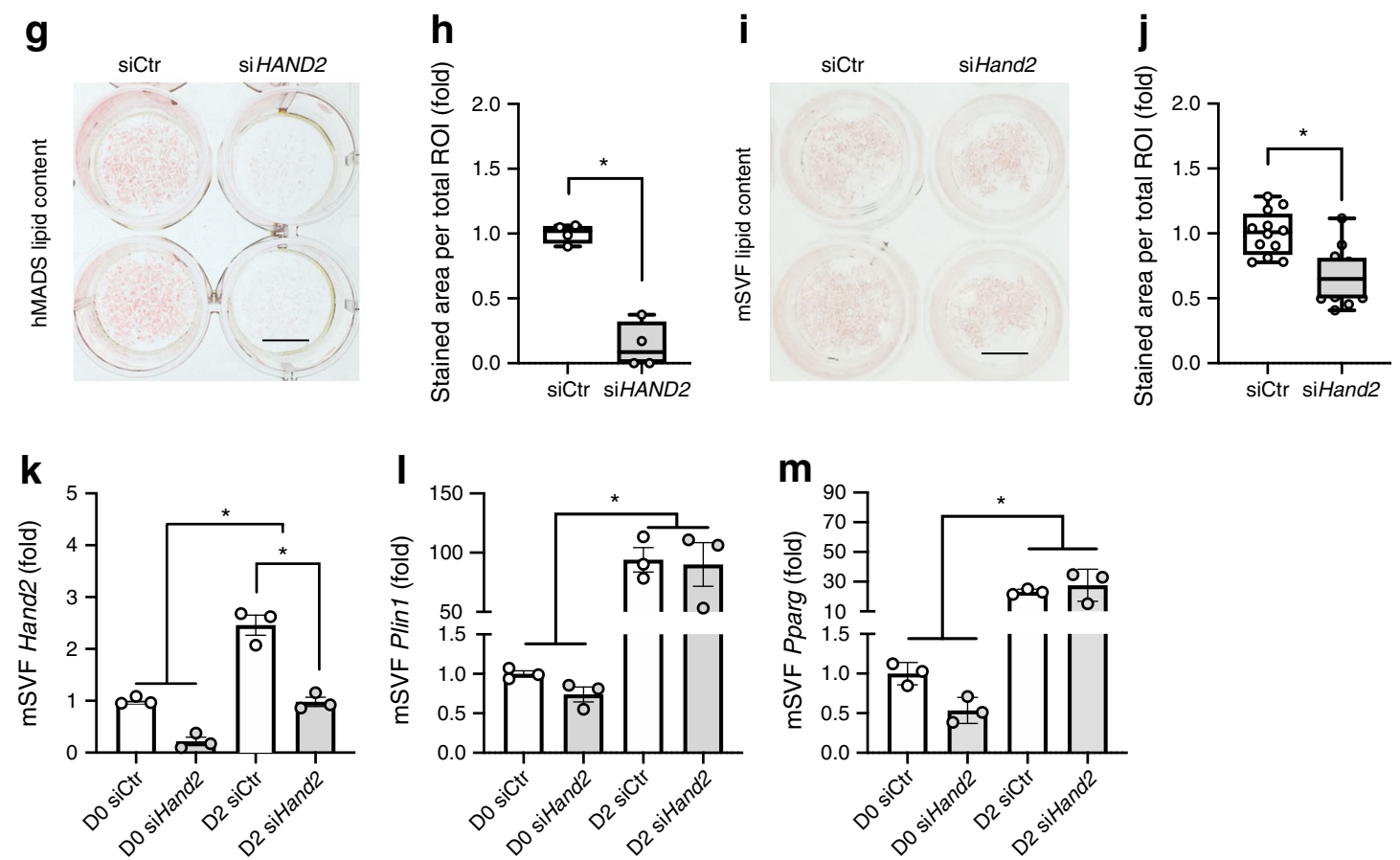

expression in mSVF transfected with siHand 22 days prior to induction and collected at day 0 and day 2 following the induction. Data were presented as fold change compared with the condition D0/siCtr (b, k$\mathbf{m})$; to the condition $\operatorname{siCtr}(\mathbf{d}-\mathbf{f}, \mathbf{h})$. Statistics: two-way ANOVA with Tukey test $(\mathbf{b}, \mathbf{k}-\mathbf{m})$, two-tailed unpaired $t$ test $(\mathbf{d}-\mathbf{f}, \mathbf{h}, \mathbf{j})$; median with upper and lower quartile \pm maximum and minimum $(\mathbf{b}, \mathbf{d}, \mathbf{e}, \mathbf{f}, \mathbf{h}, \mathbf{j})$; mean \pm SEM $(\mathbf{k}-\mathbf{m})$. Statistical significance is indicated by $* p<0.05$. Ctr, control; D, day; ORO, Oil Red O; ROI, region of interest

scWAT and BAT (ESM Fig. 2a,f,k). We also analysed the expression of $\mathrm{Nr} 3 \mathrm{cl}$, Prefl (also known as Dlk1), Plin1 and Adipo $q$ as markers of different stages of adipocyte differentiation (ESM Fig. 2b-e, g-j, 1-o). Interestingly, chronic 

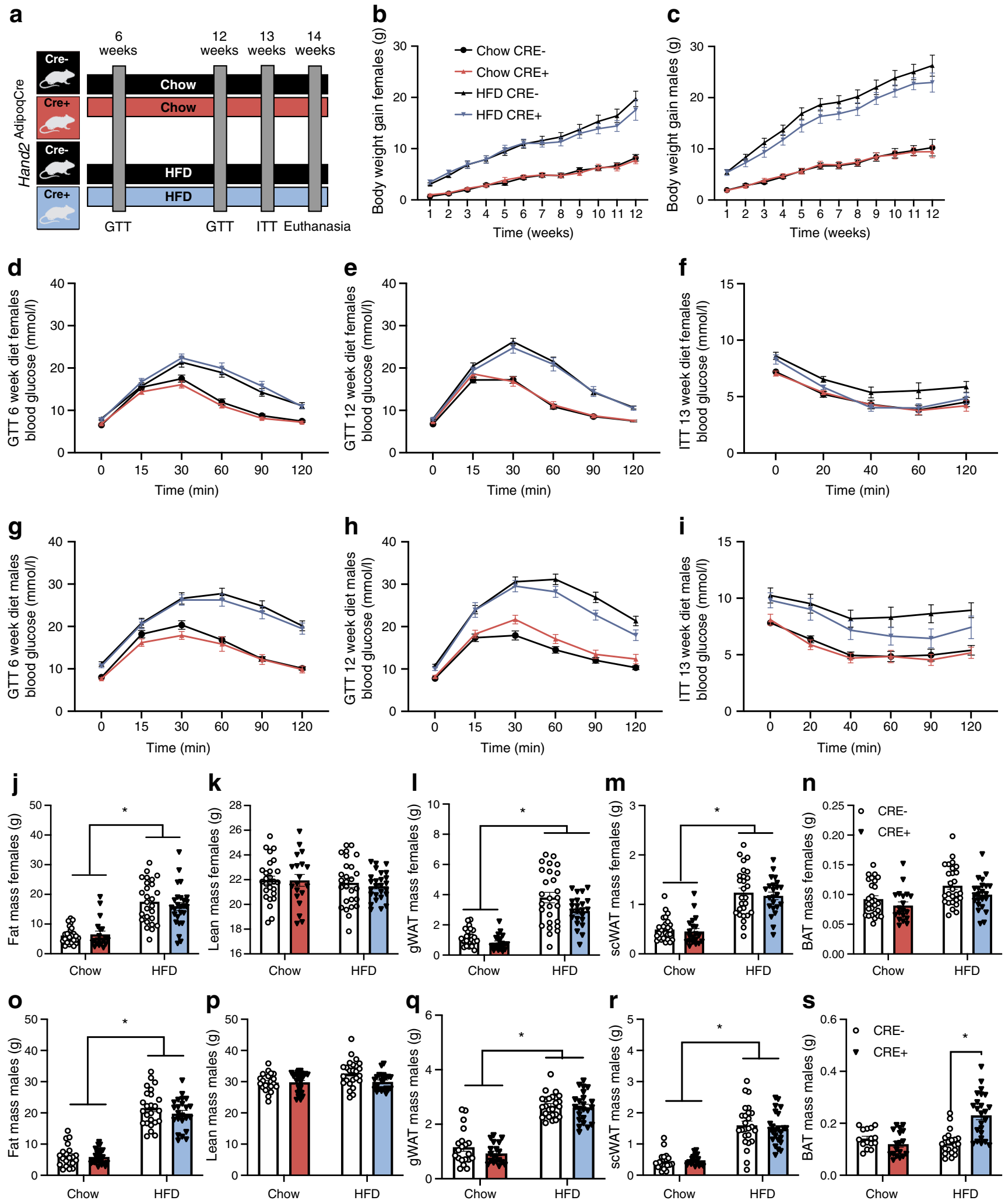

rosiglitazone treatment, which induces thermogenic adipocyte differentiation [3], did not affect Hand2 expression in mouse cells (Fig. 2d). In adipocytes differentiated from hSVF, $H A N D 2$ was higher in the classical white compared with the rosiglitazone-induced thermogenic differentiation regimen. $U C P 1$ expression was higher in thermogenic adipocytes, as expected (Fig. 2e,f). The hMADS cell model has been described as a reliable tool for studying the metabolism of 
4 Fig. 4 Metabolic phenotyping of Hand2 $2^{\text {AdipoqCre }}$ mice fed an HFD. (a) Hand $2^{\text {AdipoqCre }}(\mathrm{CRE}+)$ and wild-type (CRE-) littermates, females and males, were fed an HFD (60\%) for 6 or 12 weeks. Several metabolic variables were measured. (b, c) Body weight gain in females (b) and males (c). (d-i) GTT in females (d) and males (g) after 6 weeks of HFD diet; GTT and ITT in females $(\mathbf{e}, \mathbf{f})$ and males $(\mathbf{h}, \mathbf{i})$ after 12 weeks of HFD diet. (j-s) Final measurement of body composition $(\mathbf{j}, \mathbf{k}$, $\mathbf{o}, \mathbf{p})$ and adipose depot weight in females $(\mathbf{l}-\mathbf{n})$ and males $(\mathbf{q}-\mathbf{s})$. Each variable has been measured in three cohorts in females (Chow CRE$n=27$ mice, Chow CRE $+n=20$ mice, HFD CRE $-n=27$ mice, HFD CRE $+n=24$ mice) and males (Chow CRE $-n=21$ mice, Chow CRE+ $n=26$ mice, HFD CRE $-n=25$ mice, HFD CRE $+n=23$ mice). Statistics: two-way ANOVA with Tukey test; mean \pm SEM (b-s). Statistical significance is indicated by $* p<0.05$

white and thermogenic adipocytes [19, 20]. In hMADS cells, HAND2 was also higher in the white compared with the thermogenic differentiation regimen (Fig. 2g,h). Furthermore, we confirmed that HAND2 mRNA was induced early in hMADS adipogenesis (Fig. 2i). Interestingly, fully differentiated hMADS adipocytes showed a slightly higher expression of HAND2 than pre-adipocytes (Fig. 2k). We confirmed the early induction of Hand2 in mSVF (Fig. 2m) but could not find major change in Hand2 gene expression between undifferentiated and differentiated cells (Fig. 2o). PLIN1 was used as marker for mature adipocytes and showed higher level in differentiated adipocytes (Fig. $2 \mathrm{j}, 1, n, p)$. On the contrary, $P R E F 1$, a marker of the pre-adipocyte stage was not expressed in hMADS but showed higher levels in undifferentiated mSVF (ESM Fig. 2p,q). In summary, these data illustrate that HAND2 is highly and selectively expressed in white adipocytes with a spike of expression during the commitment phase towards the adipocyte lineage.

Loss of HAND2 impairs adipogenesis As HAND2 was induced in early adipogenesis in human and mouse adipocyte models, we hypothesised that HAND2 might be an important component of the adipogenesis program. To test this hypothesis, we silenced HAND2 gene expression in hMADS cells before the induction of differentiation (Fig. 3a). HAND2-specific siRNA treatment led to diminished levels of HAND2 mRNA at day 0 and day 2 compared with control siRNA-treated cells (Fig. $3 b)$. Transcriptomic analysis at day 2 revealed that silencing of HAND2 was associated with a marked downregulation of the expression of prominent mature adipocyte genes, including ADIPOQ, APOE, LIPE and PLIN1 (Fig. 3c). Furthermore, key adipogenic transcription factors such as PPARG, CEBPA and PPARGC1B were expressed at lower levels (Fig. $3 \mathrm{~d}-\mathrm{f}$ ), indicating that HAND2 was required for proper execution of adipogenesis. Ingenuity pathway analysis confirmed that silencing of HAND2 led to a broad dysregulation of the transcriptional programs required for adipocyte biology (ESM Fig. 3a,b). Interestingly, among the in silico predicted inhibited upstream regulators were $N R 3 C 1$ (encoding the
GR), KLF15 and PPARG, all well-known master regulators of adipogenesis. In contrast, upstream regulators associated with proliferation such as $F G F 2, T G F B$ (also known as $T G F B 1$ ) or $M I F$ were predicted to be activated (ESM Fig. $3 \mathrm{c})$. The aberrant execution of the transcriptional adipogenesis program was also mirrored in the overall cellular phenotype, as early HAND2 silencing completely abolished the differentiation of hMADS cells into mature adipocytes as demonstrated by the lack of lipid droplet formation (Fig. 3g,h). Moreover, in mSVF-derived pre-adipocytes, early silencing of Hand 2 led to lower lipid droplet formation while the induction of key adipogenic genes, including Plin1 and Pparg, remained largely intact (Fig. 3i-m). Taken together, our results indicate that HAND2 was required for adequate differentiation in human adipose-derived mesenchymal stem cells and modulated differentiation in mouse pre-adipocytes.

Hand 2 in mature adipocytes is dispensable in vivo In order to assess whether HAND2 might also be required for adipocyte function and systemic metabolic control in vivo, we created a conditional Cre-loxP mouse model for adipocyte-specific deletion of Hand2. We used an established transgenic mouse model, in which critical parts of the Hand2 gene are flanked by loxP sites [24] and crossed this model with mice carrying Cre driven by the Adipoq promoter [26], which led to genetic deletion of Hand2 in mature adipocytes in vivo (Hand2 ${ }^{\text {AdipoqCre }}$ ) (ESM Fig. 4a,b). As adipocyte function is an important pillar of lipid homeostasis during fasting and the postprandial phase, we first tested plasma triacylglycerol and non-esterified fatty acid levels in chow-fed male and female mice. Fasted and refed plasma lipid levels remained largely unchanged between Hand $2^{\text {AdipoqCre }}$ and wild-type littermate controls (ESM Fig. 4c-f). These observations were supported with human metabolic data showing no correlation between HAND2 expression in WAT and plasma triacylglycerol levels (ESM Table 2). In addition, adipocytes are critically important for sustaining metabolic control in states of caloric excess, so we next tested the role of adipocyte HAND2 in DIO mice. Both male and female mice were placed on HFD for 12 weeks to induce weight gain and insulin resistance whereas chow diet-fed mice were included as controls (Fig. 4a). As expected, HFD feeding induced markedly higher weight gain compared with chow diet in both sexes, whereas the absence of Hand 2 in white adipocytes did not have an effect (Fig. 4b,c). During the study, we performed GTTs after 6 and 12 weeks as well as an ITT after 12 weeks of the feeding regimen. As expected, HFD-fed mice displayed markedly lower glucose tolerance as well as markedly higher insulin resistance relative to chow-fed controls at both time points, however, we did not detect any genotype-specific differences (Fig. 4d-i). At the end of the study, while lean mass remained unchanged across diets and genotypes, HFD-fed mice displayed markedly higher adiposity and WAT depot weights than chow-fed 

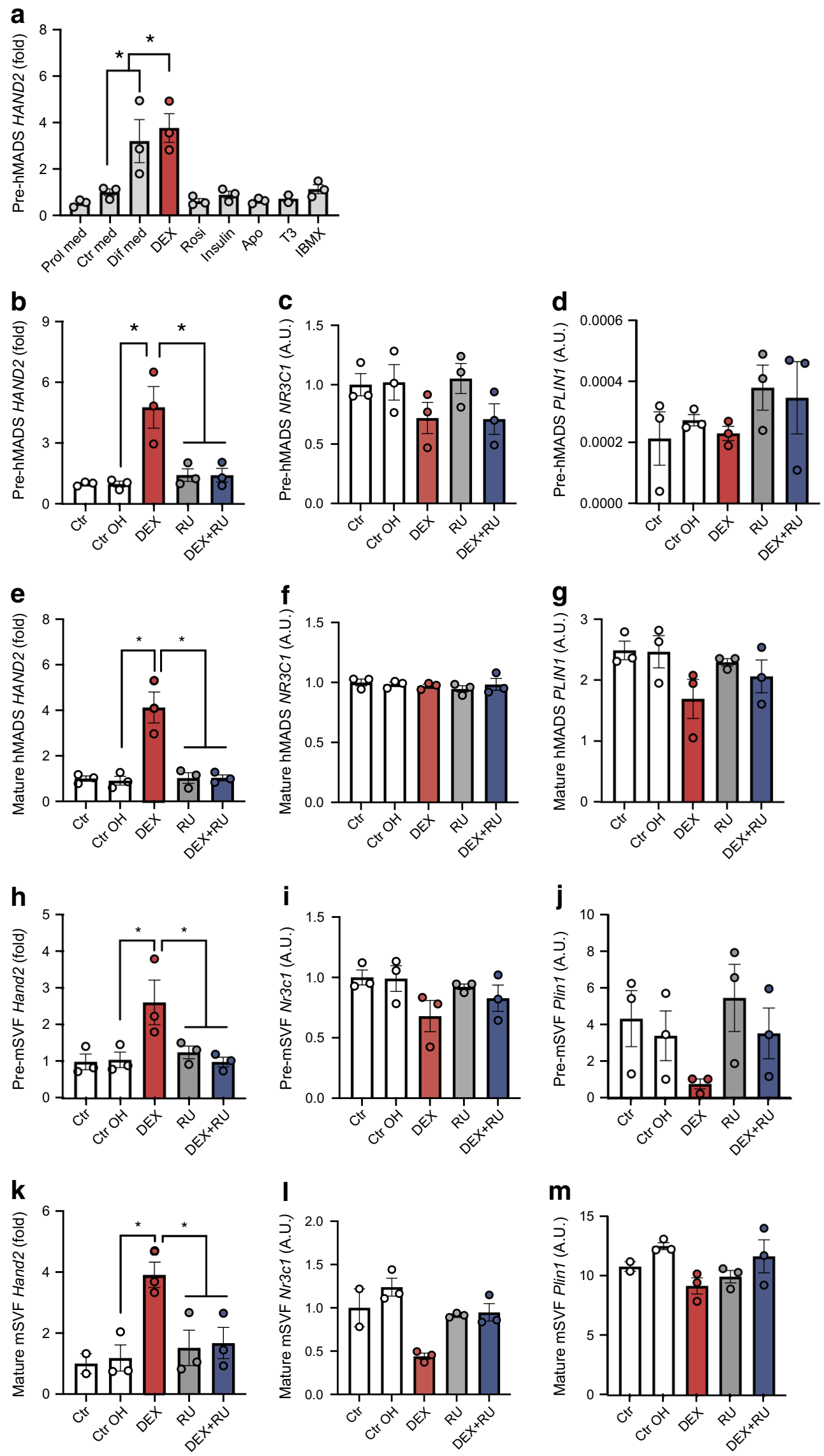
4 Fig. 5 DEX regulates HAND2 expression. (a) HAND2 expression in hMADS pre-adipocytes. Proliferation media (DMEM 10\% serum), control media (DMEM + F12), differentiation media (DMEM + F12 + $1 \mu \mathrm{mol} / 1 \mathrm{DEX}+100 \mathrm{nmol} / 1$ rosiglitazone $+10 \mathrm{nmol} / 1$ insulin $+10 \mu \mathrm{g} / \mathrm{ml}$ apotransferrin $\left.+0.2 \mathrm{nmol} / 1 \mathrm{~T}_{3}+500 \mu \mathrm{mol} / 1 \mathrm{IBMX}\right)$, rosiglitazone (DMEM + F12 + $100 \mathrm{nmol} / 1$ rosiglitazone), DEX (DMEM + F12 + 1 $\mu \mathrm{mol} / \mathrm{l} \mathrm{DEX})$, insulin (DMEM $+\mathrm{F} 12+10 \mathrm{nmol} / \mathrm{l}$ insulin), apo-transferrin $(\mathrm{DMEM}+\mathrm{F} 12+10 \mu \mathrm{g} / \mathrm{ml}$ apotransferrin $), \mathrm{T}_{3}(\mathrm{DMEM}+\mathrm{F} 12+0.2$ $\left.\mathrm{nmol} / 1 \mathrm{~T}_{3}\right), \operatorname{IBMX}(\mathrm{DMEM}+\mathrm{F} 12+500 \mu \mathrm{mol} / 1 \mathrm{IBMX})(n=3$ replicates). (b-g) HAND2, NR3C1 and PLIN1 expression in hMADS pre-adipocytes ( $n=3$ replicates) (b-d) and mature adipocytes $(n=3$ replicates) $(\mathbf{e}-\mathbf{g})$, treated with $2 \%$ ethanol, with DEX $(1 \mu \mathrm{mol} / \mathrm{l})$ and/or RU-486 $(2 \mu \mathrm{mol} / \mathrm{l})$ for $12 \mathrm{~h}(n=3$ replicates). (h-m) Hand2, $\mathrm{Nr} 3 \mathrm{cl}$ and Plin1 expression in mSVF pre-adipocytes $(n=3$ replicates) $(\mathbf{h}-\mathbf{j})$ and mature adipocytes $(n=3$ replicates) $(\mathbf{k}-\mathbf{m})$, treated with DEX (1 $\mu \mathrm{mol} / \mathrm{l})$ and/or RU-486 $(2 \mu \mathrm{mol} / \mathrm{l})$ for $12 \mathrm{~h}$. Data are presented as fold change compared with the condition Ctr med (a), to the condition $\mathrm{Ctr}$ (b, $\mathbf{e}, \mathbf{h}, \mathbf{k})$. Data are presented as arbitrary units representing copy number normalised to $T B P(\mathbf{c}, \mathbf{d}, \mathbf{f}, \mathbf{g}, \mathbf{i}, \mathbf{j}, \mathbf{l}, \mathbf{m})$. Statistics: one-way ANOVA with Tukey test; mean \pm SEM. Statistical significance is indicated by $* p<$ 0.05. Apo, apo-transferrin; Ctr, control; $\mathrm{Ctr} \mathrm{OH}, 2 \%$ ethanol; Dif, differentiation; IBMX, isobutylmethylxanthine; med, media; Rosi, rosiglitazone; RU, RU-486

controls and these differences were independent of sex and genotype (Fig. 4j-s). A careful analysis of adipose tissue histology revealed the expected increase in adipocyte diameter in WAT upon HFD feeding, but overall WAT and BAT were phenotypically similar when comparing genotypes (ESM Fig. $5 \mathrm{a}-\mathrm{f})$. Following up on these in vivo findings, we also confirmed that HAND2 is dispensable in differentiated hMADS cells in vitro as silencing of HAND2 has no effect on lipid release (ESM Fig. 6a), glucose uptake (ESM Fig. 6b) or expression of key players of metabolic pathways (ESM Fig. $6 \mathrm{c}-\mathrm{m})$ or on phosphorylation of Akt under insulin stimulation (ESM Fig. 6n). In summary, while HAND2 was required for proper adipogenesis in vitro, its silencing in vitro and in vivo in fully differentiated adipocytes did not affect the response to insulin stimulation or adipocyte function on glucose transport and lipolysis.

HAND2 is regulated by GCs via the GR Our results supported the hypothesis that HAND2 expression levels in adipocytes were determined early during adipogenesis, and that HAND2 expression was required for differentiation of stem cells into adipocytes in vitro. Among the most powerful and established inducers of adipogenesis are insulin, cAMP-raising agents (e.g. isobutylmethylxanthine [IBMX]), agonists of PPAR $\gamma$ (e.g. rosiglitazone), and GR agonists, such as DEX. As HAND2 expression was induced in cell culture upon treatment with this commonly used adipogenic hormone cocktail, we explored the ability of the individual ingredients to regulate $H A N D 2$. We found that DEX, but not the other adipogenic agents, induced HAND2 expression (Fig. 5a). This induction of HAND2 by DEX was antagonised by RU486 treatment in hMADS (Fig. 5b-g) as well as in SVF cells
(Fig. 5h-m) irrespectively of whether it was added before or after differentiation. A time course of DEX and RU-486 treatment revealed that HAND2 expression peaked after $3 \mathrm{~h}$ of treatment and remained significantly different from control cells up to at least $24 \mathrm{~h}$ (ESM Fig. 7a) NR3C1 and PLIN1 were not affected by HAND2 knockdown (ESM Fig. 7b,c). Of note, as expected, PLIN1 expression was very low in preadipocytes, and markedly higher in differentiated adipocytes, and was not significantly affected by pharmacological manipulation of GR, with the only exception being mature mSVF Hand2 $2^{\text {flox/flox }}(p<0.05)$. Prefl showed higher levels in undifferentiated mSVF (ESM Fig. 7d,e). Furthermore, in mice, a single DEX injection led to higher Hand2 expression in gWAT in females (ESM Fig. 8a,b). However, chronic DEX treatment induced an increase of energy expenditure in the light phase and a decrease in the dark phase; nevertheless no specific phenotype was observed in Hand $2^{\text {AdipoqCre }}$ mice (ESM Fig. 8c,d,g). Other metabolic variables including activity, food intake, body weight, body composition and blood glucose concentration were not influenced by the absence of Hand 2 in mature adipocytes, neither at $22^{\circ} \mathrm{C}$ nor at $30^{\circ} \mathrm{C}$ thermoneutrality (ESM Fig. $8 \mathrm{e}, \mathrm{f}, \mathrm{h}-\mathrm{m})$. Interestingly, chronic DEX treatment by daily injections for 2 days or 2 weeks lowered Hand 2 levels in gWAT (ESM Fig. 8n,o). Similar findings were observed in humans receiving daily DEX treatment for more than a week for chronic inflammatory diseases. (ESM Fig. 8p). Congruent with a GR-dependent mechanism, siRNAmediated knockdown of NR3C1 in hMADS pre-adipocytes as well as mature adipocytes (Fig. 6a-d) abolished the effect of DEX on HAND2. Genetic inactivation of Hand2 in SVF from $\mathrm{gWAT}$ of Hand $2^{\text {flox/flox }}$ mice with Cre-encoding mRNA both in pre-adipocytes and mature adipocytes prevented Hand2 increase in pre-adipocytes (condition $\mathrm{GFP}+\mathrm{DEX}$ vs CRE + DEX $p=0.059)$ and mature adipocytes (condition GFP + DEX vs CRE + DEX $p=0.11$ ) under DEX treatment but did not affect GR expression (Fig. 6e-h). In pre-adipocytes and mature adipocytes isolated from WAT SVF of $N r 3 c 1^{\text {flox/flox }}$ mice crossed with mice expressing the global tamoxifen-inducible Cre-ERT2 fusion protein $\left(G R^{\text {ERT2CRE }}\right)$ [25], we confirmed that GR regulated Hand2 expression as tamoxifen treatment abrogated the increase in Hand2 under DEX stimulation (Fig. 6i-1). Using an RNA interference approach in mSVF we made similar observations (condition siCtr + DEX vs siHand + DEX $p=0.09$ ) (Fig. 6m-n). Furthermore, in all the conditions described above, as expected, PLIN1 expression was almost nonexistent in pre-adipocytes compared with adipocytes and remained unaffected by the different treatments while Pref1 expression was higher in undifferentiated cells (ESM Fig. $7 \mathrm{f}-\mathrm{p}$ ). Altogether these data support the notion that HAND2 is regulated by the $\mathrm{GC}-\mathrm{GR}$ pathway during adipocyte differentiation. 

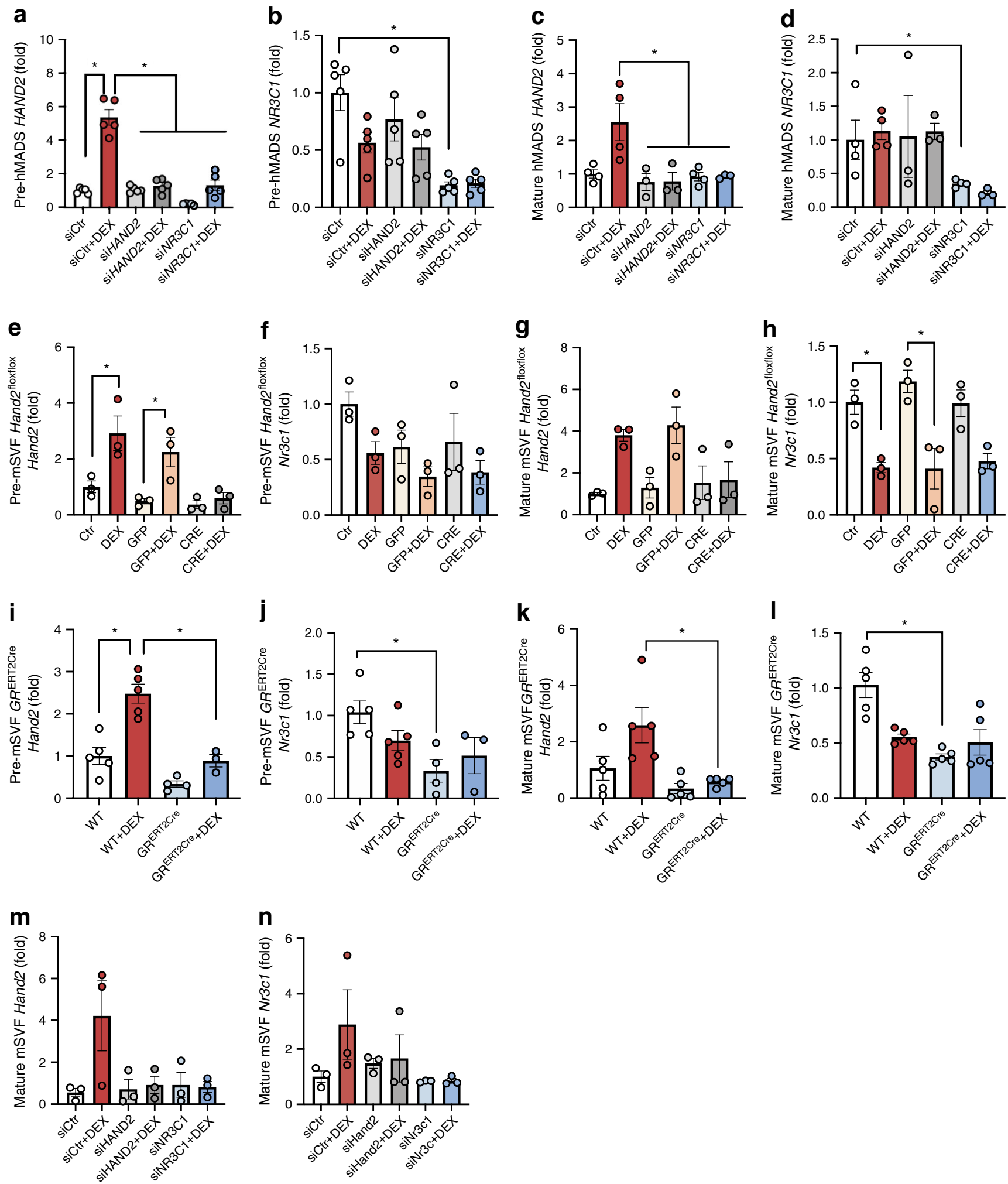

Hand 2 expression is necessary but insufficient for adipocyte differentiation We explored whether increasing Hand 2 expression was sufficient to compensate for GCs at the beginning of differentiation. To this end, we overexpressed Hand2 using a pcDNA-3XFlag-Hand 2 vector $24 \mathrm{~h}$ before inducing

the differentiation of 3T3-L1 cells either using a classical cocktail of induction including DEX $(1 \mu \mathrm{mol} / \mathrm{l})$ or with a cocktail depleted of DEX. Comparing cells transfected with the control or Hand 2 vectors, we did not detect any significant difference in key differentiation markers, neither $24 \mathrm{~h}$ nor 6 
Fig. 6 HAND2 is regulated by GCs via the GR. (a-d) HAND2 and $N R 3 C 1$ expression in hMADS pre-adipocytes $(n=5$ replicates) $(\mathbf{a}, \mathbf{b})$ and mature adipocytes $(n=4$ replicates) $(\mathbf{c}, \mathbf{d})$, transfected with siHAND2 or siNR3C1, treated or not with DEX $(1 \mu \mathrm{mol} / \mathrm{l})$ for $12 \mathrm{~h}$. (eh) Hand 2 and $\mathrm{Nr} 3 \mathrm{cl}$ expression in mSVF pre-adipocytes $(n=3$ replicates) (e,f) and mature adipocytes $(n=3$ replicates) $(\mathbf{g}, \mathbf{h})$ from Hand $2^{\text {flox/flox }}$ mice. (i-I) Hand 2 and $N r 3 c l$ expression in MSVF in preadipocytes ( $n=3-5$ replicates) $(\mathbf{i}, \mathbf{j})$ and differentiated adipocytes $(n=5$ replicates) $(\mathbf{k}, \mathbf{l})$, all treated with tamoxifen and treated or not with DEX (1 $\mu \mathrm{mol} / \mathrm{l})$ for $12 \mathrm{~h}$ from $G R^{\text {ERT2Cre }}$ mice. (m,n) Hand2 and $\mathrm{Nr} 3 \mathrm{cl}$ expression in $\mathrm{mSVF}$ differentiated adipocytes and transfected with siHand 2 or siNr3cl $24 \mathrm{~h}$ before DEX treatment $(1 \mu \mathrm{mol} / \mathrm{l})$ for $12 \mathrm{~h}$. Data are presented as fold change compared to the condition $\operatorname{siCtr}(\mathbf{a}-\mathbf{d}$, $\mathbf{m}, \mathbf{n})$; to the condition $\mathrm{Ctr}(\mathbf{e}-\mathbf{h})$, to the condition WT (i-l). Statistics: twoway ANOVA with Tukey test; mean \pm SEM $(\mathbf{a}-\mathbf{n})$. Statistical significance is indicated by $* p<0.05$, WT, wild-type

days after induction (ESM Fig. 9a-h, k-q) and lipid accumulation was unchanged (ESM Fig. 9i-j). In conclusion, while HAND2 is required early in the transition of mesenchymal cells into the adipocyte lineage, its induction is insufficient for differentiation and unable to compensate for $\mathrm{GC}$ stimulation.

Gene networks regulated by the GR-HAND2 pathway Our results indicate that HAND2 was induced by GR and required for adipocyte differentiation but dispensable in mature adipocytes. Therefore, we set out to understand the transcriptional networks during early adipogenesis downstream of the GRHAND2 pathway. We silenced HAND2 and NR3C1 in hMADS cells and treated the cells 2 days later with DEX for $12 \mathrm{~h}$ and performed RNAseq (Fig. 7a-c). While the transcriptome signature of cells with $\mathrm{NR} 3 \mathrm{C} 1$ silencing treated with DEX clustered together with the control samples without DEX treatment (Fig. 7d), cells with HAND2 silencing clustered with control treated with DEX, suggesting that HAND2 regulates only a small subset of GR-related genes. Using a gene set enrichment analysis [27, 28], we determined the transcriptional networks regulated by GC-GR signalling for benchmarking our dataset. Indeed, we found many confirmed GC-GR signalling pathways, underlining the reliability of our dataset (Fig. 7e). Focusing on gene expression levels that were differentially and commonly regulated by GR and HAND2 as part of the GR-HAND2 pathway we found four main pathways to be regulated: the metapathway biotransformation phase I and II, including genes from the cytochrome p450 family implicated in the synthesis of cholesterol steroids and other lipids, the mammalian target of rapamycin (mTOR) pathway and the large family of class A rhodopsin-like G protein-coupled receptors (GPCRs) and the signalling pathway vascular endothelial growth factor (VEGF)A-VEGFR2 (Fig. 7f). We confirmed the regulation of several of these gene sets in gWAT of Hand2 ${ }^{\text {AdipoqCre }}$ mice including Efnal, Efna2, Fmo2 and Cyp2f1 (ESM Fig. 10a). Using WAT SVF from Hand $2^{3 \text { XFlag }}$ mice or the associated vector pcDNA-3XFlag-
Hand2 to overexpress HAND2 in 3T3-L1 cells, we investigated by ChIP qPCR whether HAND2 was directly binding putative targets that we selected from our RNASeq data (Efna2, Clnd1, Rgs 3 and Rheb), known targets of GR (Perl and Gilz [also known as Tsc22d3] or Tbx2 and Hand2 itself, which were already published to be targets of HAND2 [15]. We did not find evidence that HAND2 was enriched in the selected binding sites (ESM Fig. 10b-g). In summary, these data confirm that HAND2 plays an important role very early in the adipocyte differentiation process and might play an important role in the execution of the GC-GR program.

\section{Discussion}

In this study, we show that HAND2 is a critical factor for early adipogenesis, regulated by GC-GR signalling and correlated to body weight and obesity in mice and humans. In hMADS cells, the earlier we manipulated HAND2 expression, the more severe was the effect in interfering with proper adipogenesis, while in mSVF the effects were similar but much smaller in magnitude. These differences could be explained by the different level of commitment of hMADS compared with primary pre-adipocytes. Furthermore, while GCs are required for human adipocyte differentiation they are largely dispensable in mouse models [11]. Therefore, it should not come as a surprise that our conditional Hand 2 mouse model using Adipoq-Cre did not show any overt metabolic phenotype, as the Adipoq gene and hence the Cre recombinase are expressed relatively late in adipocyte differentiation [26]. One way to probe this further would be to deplete Hand 2 under a Cre driver specifically expressed in the commitment phase of the adipocytes, such as Wnt or hedgehog [29, 30]. Nevertheless, those master regulators are not specific to adipocytes and might lead to lethality in the very early stage of the embryo development similarly to the global deletion of Hand2 [16, 17]. As Handl gene expression was undetectable in adipocytes, it is unlikely that there is compensation of HAND2 loss by HAND1 activity in our studies.

The second major finding of our study is that HAND2 expression is regulated by GCs via GR during early and late stages of adipogenesis. Available data in the literature did not identify HAND2 as a direct target of GR [11, 31, 32], a finding that we confirmed by performing GR ChIP qPCR. We also investigated the HAND2 promoter area and did not find potential interactions between PPARG, PRDM16, CEBPA and $C E B P B$. Our RNAseq results demonstrated that GR is required for the global effects of DEX, which was expected. In contrast, cells with loss of HAND2 still had a relatively intact transcription profile, indicating that HAND2 only regulates a small and defined set of genes upon DEX treatment. Further analyses are required to interrogate the relevance of these putative downstream effectors of HAND2 for 
a

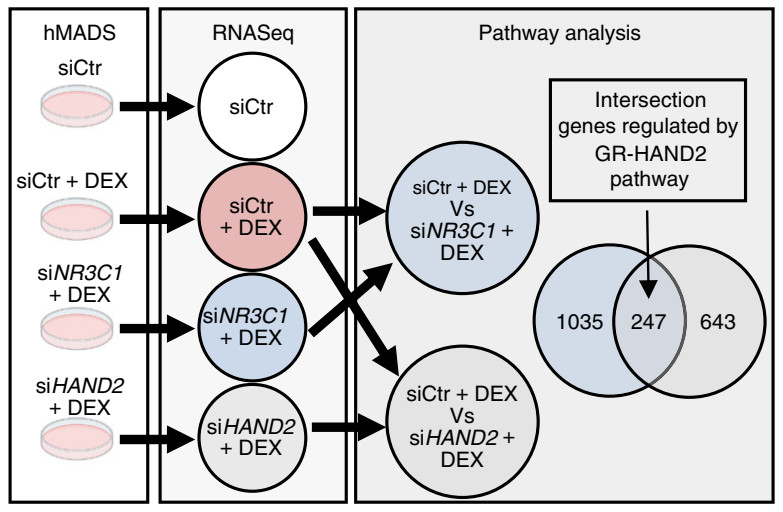

d
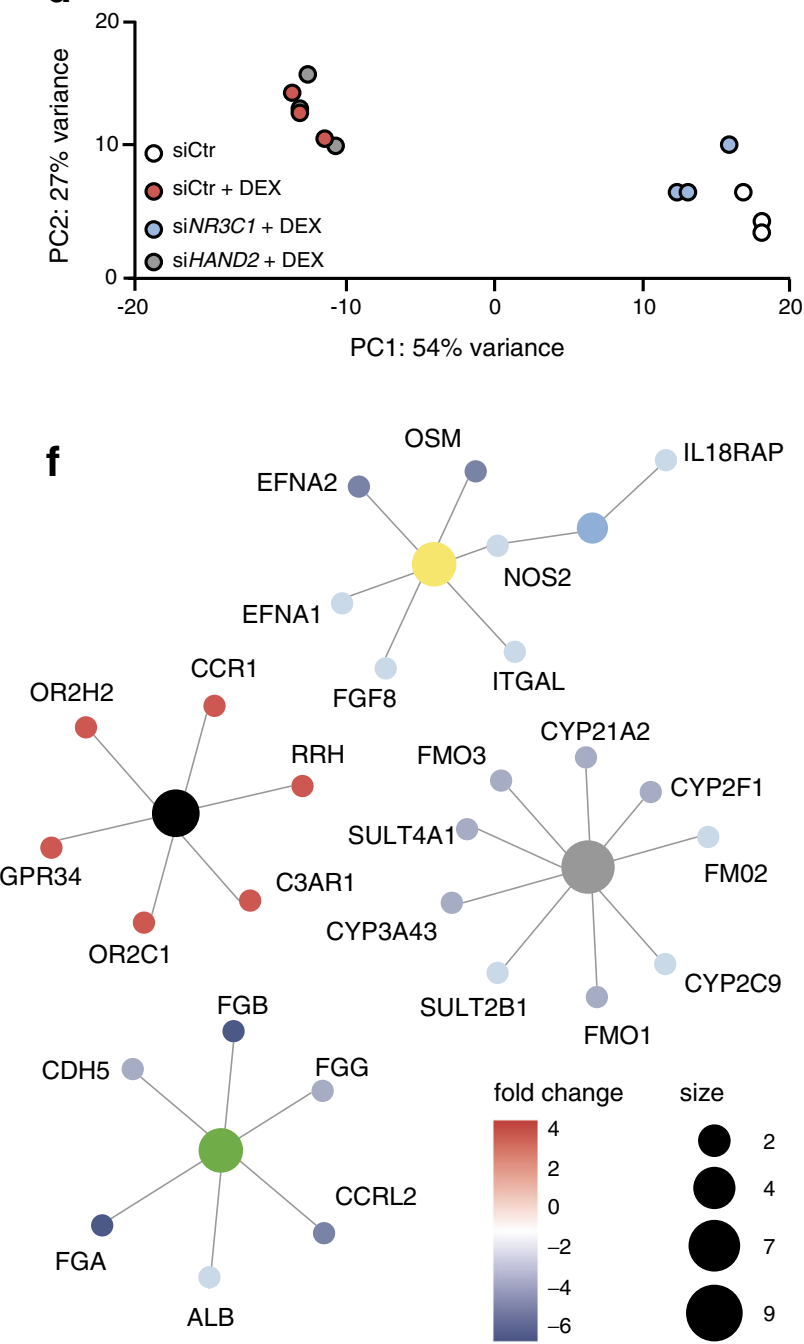

Focal adhesion-PI3k-Akt-mTOR-signalling pathway

- GPCRs, class A rhodopsin-like

- IL-18 signalling pathway

- Metapathway biotransformation phase I and II

- VEGFA-VEGFR2 signalling pathway b

C
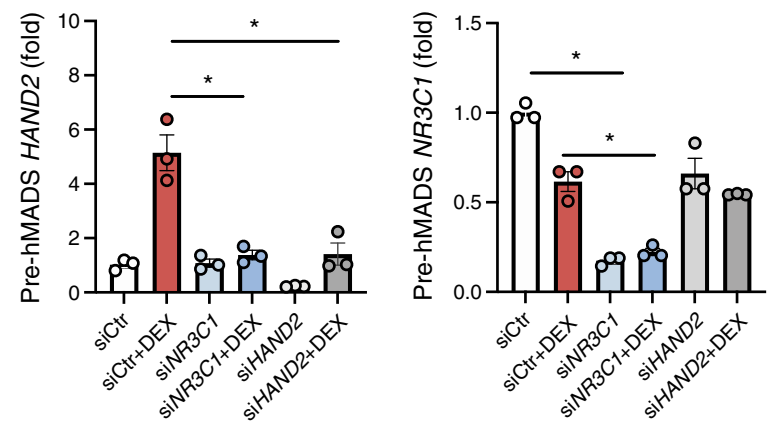

e

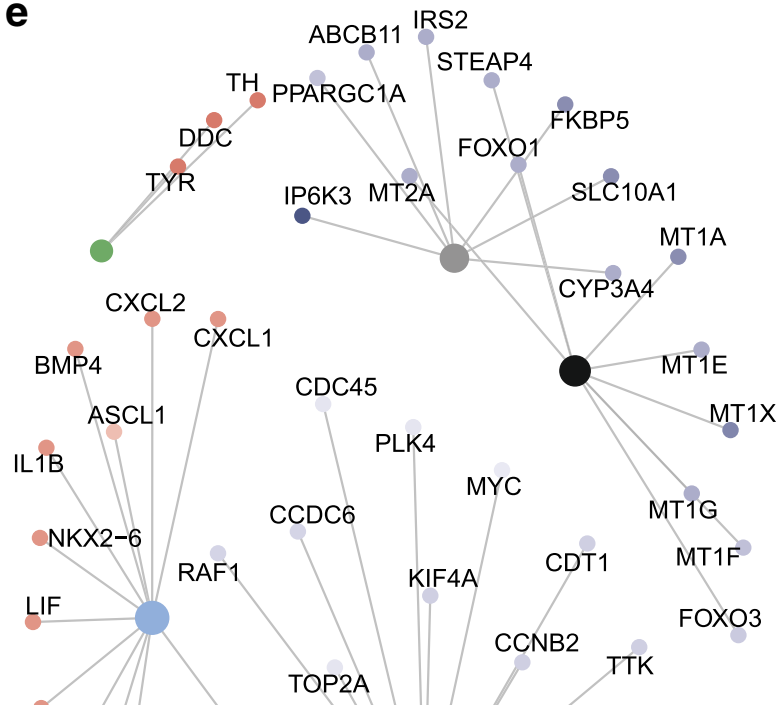

FGF2

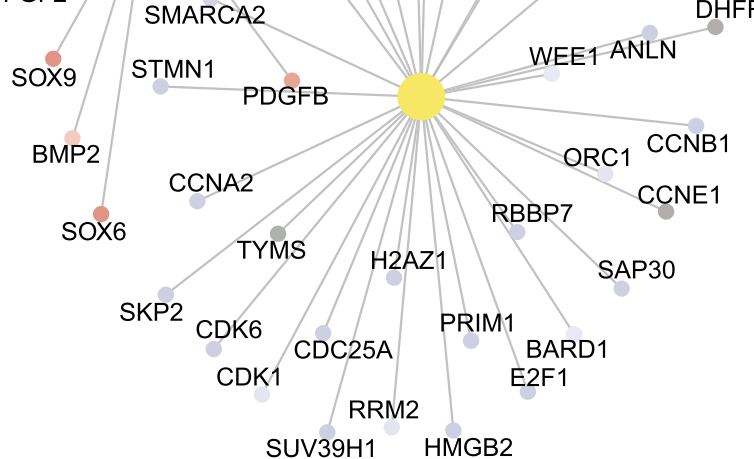

\footnotetext{
fold change size

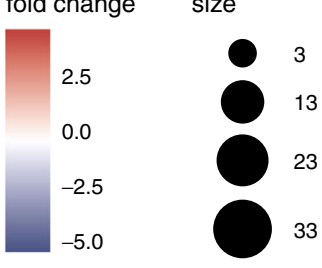

- Dopamine metabolism

- Farnesoid $\mathrm{X}$ receptor pathway

- Oligodendrocyte specification and differentiation

Retinoblastoma gene in cancer
} 
Fig. 7 Gene networks during adipocyte differentiation regulated by the GR-HAND2 pathway. (a) Experimental protocol: hMADS preadipocytes were transfected with siNR3Cl or siHAND2 $36 \mathrm{~h}$ prior to a $12 \mathrm{~h}$ treatment with DEX $(1 \mu \mathrm{mol} / \mathrm{l})$. Pathway analysis was performed on the genes regulated by $\mathrm{NR} 3 \mathrm{Cl}$ and commonly regulated by $\mathrm{NR} 3 \mathrm{Cl}$ and HAND2 ( $n=3$ replicates). (b-c) qPCR analysis of HAND2 and NR3C1 gene expression. (d) Principal component analyses of the RNAseq data. (e,f) GO pathway analysis of siCtr + DEX vs siNR3C1 + DEX (e) and of the intersection between siCtr + DEX vs siNR3Cl + DEX and siCtr + DEX vs siHAND2 + DEX (f). Data are presented as fold change compared with the condition $\mathrm{siCtr}(\mathbf{b}, \mathbf{c})$. Statistics: two-way ANOVA with Tukey test; mean $\pm \operatorname{SEM}(\mathbf{b}, \mathbf{c})$. Statistical significance is $* p<$ 0.05. GPCR, G protein-coupled receptors; mTOR, mammalian target of rapamycin; PC, principal component; PI3K, phosphatidylinositol 3kinase; VEGF, vascular endothelial growth factor

adipogenesis. Considering the pleiotropic effects of DEX and GR signalling in the body, it is possible that some of the previous functions attributed to HAND2, for example in the heart, also involve GR activity as well as, vice versa, that established GR-mediated effects, for example in immune cells, could also involve HAND2.

Finally, lower levels of Hand2 in gWAT and visWAT observed under chronic DEX treatment might be explained by the induction of a regulatory feedback loop or by the length of the exposure. Furthermore, HAND2 expression correlates with BMI in visWAT but not scWAT. While more work is needed to understand this depot-specific regulation, it might relate to $11 \beta$-hydroxysteroid dehydrogenase (HSD)-1, a key enzyme in GC metabolism that contributes to the increased levels of GCs specifically in visWAT of obese individuals [33-35]. Under these conditions, reactivation of HAND2 expression in obesity could help stimulate adipogenesis and healthy adipose tissue expansion, thus restoring insulin sensitivity and metabolic health. In summary, our study introduces HAND2 as a novel player in adipogenesis and highlights a new layer of GC-GR signalling, thus enhancing our understanding of adipocyte biology in obesity.

Supplementary Information The online version contains peer-reviewed but unedited supplementary material available at https://doi.org/10.1007/ s00125-021-05470-y.

Acknowledgements We thank E.Z. Amri (Institut de Biologie Valrose, Université Nice Sophia Antipolis, France) for providing hMADS cells, as well as R. Zeller and A. Zuniga (Developmental Genetics, Department of Biomedicine, University of Basel, Switzerland) for providing the Hand $2^{\text {flox/flox }}$ mice, Hand $2^{3 \text { XFlag }}$ mice and the pcDNA-3XFlag-Hand 2 vector. We thank A. Loft, A. Georgiadi and P. Morigny (Institute for Diabetes and Cancer, Helmholtz Center Munich, Germany) for discussion and M. Bamberger (Institute of Experimental Genetics, Helmholtz Zentrum Munich, Germany) for excellent technical assistance. The graphical abstract and Figs 3a, 4a, 7a and ESM Fig. 4a were created with BioRender.com.

Data availability Array data have been submitted to the GEO database at NCBI (GSE148699). Other data that support the findings of this study are available upon reasonable request.
Funding Open Access funding enabled and organized by Projekt DEAL. MG was supported by Alexander von Humboldt Foundation postdoctoral fellowships. PF-P was supported by the Deutsche Forschungsgemeinschaft (FI 1700/7-1, Heisenberg Program). DT was supported by a grant from the German Research Association (TE912/22). JT was supported by the Deutsche Forschungsgemeinschaft (TU 220/ 13-1). HU was supported by the Deutsche Forschungsgemeinschaft Trans-Regio (TRR205) adrenal gland, and (CRC1064) chromatindynamics as well as the Deutsches Zentrum für Diabetesforschung. AB was supported by the Deutsche Forschungsgemeinschaft Sonderforschungsbereich 1123 (B10), and the Deutsches Zentrum für Herz-Kreislauf-Forschung Junior Research Group Grant. SH and JB were supported by the Helmholtz Future topic 'Aging and Metabolic Programming, AMPro'. SH was supported by the Deutsche Forschungsgemeinschaft Trans-Regio (TRR205) and the Deutsches Zentrum für Herz-Kreislauf-Forschung Standortprojekt Cardiometabolism.

Authors' relationships and activities The authors declare that there are no relationships or activities that might bias, or be perceived to bias, their work.

Contribution statement $\mathrm{MG}, \mathrm{MS}, \mathrm{AB}$ and $\mathrm{SH}$ substantially contributed to the conception or design of the work. $\mathrm{MG}, \mathrm{AB}$ and $\mathrm{SH}$ drafted the article. MG interpreted the work. All authors contributed to the acquisition and analysis of the data. All the authors revised the manuscript critically for important intellectual content and approved the final version of the manuscript. $\mathrm{MG}, \mathrm{AB}$ and $\mathrm{SH}$ are the guarantors of this work.

Open Access This article is licensed under a Creative Commons Attribution 4.0 International License, which permits use, sharing, adaptation, distribution and reproduction in any medium or format, as long as you give appropriate credit to the original author(s) and the source, provide a link to the Creative Commons licence, and indicate if changes were made. The images or other third party material in this article are included in the article's Creative Commons licence, unless indicated otherwise in a credit line to the material. If material is not included in the article's Creative Commons licence and your intended use is not permitted by statutory regulation or exceeds the permitted use, you will need to obtain permission directly from the copyright holder. To view a copy of this licence, visit http://creativecommons.org/licenses/by/4.0/.

\section{References}

1. Bartelt A, Heeren J (2014) Adipose tissue browning and metabolic health. Nat Rev Endocrinol 10(1):24-36. https://oi.org/10.1038/ nrendo.2013.204

2. Lowe CE, O'Rahilly S, Rochford JJ (2011) Adipogenesis at a glance. J Cell Sci 124(16):2681-2686. https://doi.org/10.1242/jcs. 079699

3. Petrovic N, Walden TB, Shabalina IG, Timmons JA, Cannon B, Nedergaard J (2010) Chronic peroxisome proliferator-activated receptor gamma (PPARgamma) activation of epididymally derived white adipocyte cultures reveals a population of thermogenically competent, UCP1-containing adipocytes molecularly distinct from classic brown adipocytes. J Biol Chem 285(10):7153-7164. https:// doi.org/10.1074/jbc.M109.053942

4. Buren J, Liu H, Jensen J, Eriksson J (2002) Dexamethasone impairs insulin signalling and glucose transport by depletion of insulin receptor substrate-1, phosphatidylinositol 3-kinase and protein 
kinase B in primary cultured rat adipocytes. Eur J Endocrinol 419429. https://doi.org/10.1530/eje.0.1460419

5. Zinker B, Mika A, Nguyen P et al (2007) Liver-selective glucocorticoid receptor antagonism decreases glucose production and increases glucose disposal, ameliorating insulin resistance. Metabolism 56(3): 380-387. https://doi.org/10.1016/j.metabol.2006.10.021

6. Kuo T, McQueen A, Chen T-C, Wang J-C (2015) Regulation of glucose homeostasis by glucocorticoids. In: Wang J-C, Harris C (eds) Glucocorticoid signaling. Springer New York, New York, NY, pp 99-126

7. Lee RA, Harris CA, Wang J-C (2018) Glucocorticoid receptor and adipocyte biology. Nucl Recept Res 5. https://doi.org/10.32527/ 2018/101373

8. Luijten IHN, Cannon B, Nedergaard J (2019) Glucocorticoids and brown adipose tissue: do glucocorticoids really inhibit thermogenesis? Mol Asp Med 68:42-59. https://doi.org/10.1016/j.mam.2019.07.002

9. Glantschnig C, Mattijssen F, Vogl ES et al (2019) The glucocorticoid receptor in brown adipocytes is dispensable for control of energy homeostasis. EMBO Rep 20(11). https://doi.org/10.15252/embr. 201948552

10. Bauerle KT, Hutson I, Scheller EL, Harris CA (2018) Glucocorticoid receptor signaling is not required for in vivo adipogenesis. Endocrinology 159(5):2050-2061. https://doi.org/10. 1210/en.2018-00118

11. Park Y-K, Ge K (2017) Glucocorticoid receptor accelerates, but is dispensable for, adipogenesis. Mol Cell Biol 37(2):e00260 e00216. https://doi.org/10.1128/MCB.00260-16

12. Wajchenberg BL, Bosco A, Marone MM et al (1995) Estimation of body fat and lean tissue distribution by dual energy X-ray absorptiometry and abdominal body fat evaluation by computed tomography in Cushing's disease. J Clin Endocrinol Metab 80(9):27912794. https://doi.org/10.1210/jcem.80.9.7673425

13. Keller M, Hopp L, Liu X et al (2017) Genome-wide DNA promoter methylation and transcriptome analysis in human adipose tissue unravels novel candidate genes for obesity. Mol Metab 6(1):86100. https://doi.org/10.1016/j.molmet.2016.11.003

14. Baghalishahi M, Efthekhar-Vaghefi SH, Piryaei A, NematolahiMahani SN, Mollaei HR, Sadeghi Y (2018) Cardiac extracellular matrix hydrogel together with or without inducer cocktail improves human adipose tissue-derived stem cells differentiation into cardiomyocyte-like cells. Biochem Biophys Res Commun 502(2): 215-225. https://doi.org/10.1016/j.bbrc.2018.05.147

15. Osterwalder M, Speziale D, Shoukry M et al (2014) HAND2 targets define a network of transcriptional regulators that compartmentalize the early limb bud mesenchyme. Dev Cell 31(3):345357. https://doi.org/10.1016/j.devcel.2014.09.018

16. Srivastava D, Thomas T, Lin Q, Kirby ML, Brown D, Olson EN (1997) Regulation of cardiac mesodermal and neural crest development by the bHLH transcription factor, dHAND. Nat Genet 16(2): 154-160. https://doi.org/10.1038/ng0697-154

17. McFadden DG, McAnally J, Richardson JA, Charité J, Olson EN (2002) Misexpression of dHAND induces ectopic digits in the developing limb bud in the absence of direct DNA binding. Dev Camb Engl 129(13):3077-3088

18. Vincentz JW, Barnes RM, Firulli AB (2011) Hand factors as regulators of cardiac morphogenesis and implications for congenital heart defects. Birt Defects Res A Clin Mol Teratol 91(6):485494. https://doi.org/10.1002/bdra.20796

19. Elabd C, Chiellini C, Massoudi A et al (2007) Human adipose tissue-derived multipotent stem cells differentiate in vitro and in vivo into osteocyte-like cells. Biochem Biophys Res Commun 361(2):342-348. https://doi.org/10.1016/j.bbrc.2007.06.180
20. Rodriguez A-M, Elabd C, Delteil F et al (2004) Adipocyte differentiation of multipotent cells established from human adipose tissue. Biochem Biophys Res Commun 315(2):255-263. https:// doi.org/10.1016/j.bbrc.2004.01.053

21. Pisani DF, Beranger GE, Corinus A et al (2016) The $\mathrm{K}^{+}$channel TASK1 modulates $\beta$-adrenergic response in brown adipose tissue through the mineralocorticoid receptor pathway. FASEB J 30(2): 909-922. https://doi.org/10.1096/fj.15-277475

22. Bartelt A, Widenmaier SB, Schlein C et al (2018) Brown adipose tissue thermogenic adaptation requires Nrfl-mediated proteasomal activity. Nat Med 24(3):292-303. https://doi.org/10.1038/nm.4481

23. Uhlenhaut NH, Barish GD, Yu RT et al (2013) Insights into negative regulation by the glucocorticoid receptor from genome-wide profiling of inflammatory cistromes. Mol Cell 49(1):158-171. https://doi.org/10.1016/j.molcel.2012.10.013

24. Galli A, Robay D, Osterwalder M et al (2010) Distinct roles of Hand 2 in initiating polarity and posterior Shh expression during the onset of mouse limb bud development. PLoS Genet 6(4): e1000901. https://doi.org/10.1371/journal.pgen.1000901

25. Rapp AE, Hachemi Y, Kemmler J, Koenen M, Tuckermann J, Ignatius A (2018) Induced global deletion of glucocorticoid receptor impairs fracture healing. FASEB J Off Publ Fed Am Soc Exp Biol 32(4):2235-2245. https://doi.org/10.1096/fj.201700459RR

26. Eguchi J, Wang X, Yu S et al (2011) Transcriptional control of adipose lipid handling by IRF4. Cell Metab 13(3):249-259. https://doi.org/10.1016/j.cmet.2011.02.005

27. Mootha VK, Lindgren CM, Eriksson K-F et al (2003) PGC-1alpharesponsive genes involved in oxidative phosphorylation are coordinately downregulated in human diabetes. Nat Genet 34(3):267273. https://doi.org/10.1038/ng1180

28. Subramanian A, Tamayo P, Mootha VK et al (2005) Gene set enrichment analysis: a knowledge-based approach for interpreting genome-wide expression profiles. Proc Natl Acad Sci U S A 102(43):15545-15550. https://doi.org/10.1073/pnas.0506580102

29. Harfe BD, Scherz PJ, Nissim S, Tian H, McMahon AP, Tabin CJ (2004) Evidence for an expansion-based temporal Shh gradient in specifying vertebrate digit identities. Cell 118(4):517-528. https:// doi.org/10.1016/j.cell.2004.07.024

30. Lewis AE, Vasudevan HN, O'Neill AK, Soriano P, Bush JO (2013) The widely used Wnt1-Cre transgene causes developmental phenotypes by ectopic activation of Wnt signaling. Dev Biol 379(2):229 234. https://doi.org/10.1016/j.ydbio.2013.04.026

31. Cohen DM, Won K-J, Nguyen N, Lazar MA, Chen CS, Steger DJ (2015) ATF4 licenses C/EBP $\beta$ activity in human mesenchymal stem cells primed for adipogenesis. eLife 4:e06821. https://doi. org/10.7554/eLife.06821

32. Steger DJ, Grant GR, Schupp M et al (2010) Propagation of adipogenic signals through an epigenomic transition state. Genes Dev 24(10):1035-1044. https://doi.org/10.1101/gad.1907110

33. Doig CL, Fletcher RS, Morgan SA et al (2017) $11 \beta$-HSD1 modulates the set point of brown adipose tissue response to glucocorticoids in male mice. Endocrinology 158(6):1964-1976. https://doi. org/10.1210/en.2016-1722

34. Liu J, Kong X, Wang L et al (2013) Essential roles of 11 $\beta$-HSD1 in regulating brown adipocyte function. J Mol Endocrinol 50(1):103113. https://doi.org/10.1530/JME-12-0099

35. Masuzaki H (2001) A transgenic model of visceral obesity and the metabolic syndrome. Science 294(5549):2166-2170. https://doi. org/10.1126/science. 1066285

Publisher's note Springer Nature remains neutral with regard to jurisdictional claims in published maps and institutional affiliations. 